\title{
Study on Strength, Deformation and Failure Properties of Hollow Cylindrical Rock Specimens
}

\author{
GOU Yong ${ }^{a}$, PEI Hongyan ${ }^{b}$, WU Zhenhua ${ }^{c}$ \\ School of Civil Engineering and Architecture, Hubei Polytechnic University, Huangshi Hubei, \\ 435003, China \\ aemail:109075425@qq.com, bemail: 568578496@qq.com, cemail: 47261571@qq.com
}

\begin{abstract}
Keywords: rock mechanics; hollow specimen; marble; strength and deformation; failure Abstract: In order to simulate the influence of excavation on the whole strength, deformation and failure of rock mass, uniaxial and pseudo-triaxial compression tests on solid and hollow cylinder specimens of marble without inner pressure were carried out with servo-controlled rock testing machine. The results show that the existence of the hollow has little influence on the uniaxial compressive strength and deformation of the rock, and the internal stress of the specimens tend to be uniformly distributed, resulting in a single section shear slip failure; In the pseudo-triaxial compression test, the strength of the specimen increases linearly with the confining pressure. The existence of the hollow makes the free surface inside the specimen, which contributes to the occurrence of local failure. During the compression process, the material near the hollow first yielded, the aperture was expanding, the effective bearing area decreased, the stress non-uniform distribution, the bearing capacity decreased, the peak deformation of the tunnel specimen was significantly lower than the solid specimen, resulting in the stepped surface.
\end{abstract}

\section{Introduction}

Many rock engineering involves rock excavation, such as tunnel development and underground mining and other projects, especially at present with the depth of coal mining continues to increase, wellbore and roadway force is quite complex, its deformation and damage become quite serious, in addition Its maintenance work is very difficult. If carry out experimental research before the construction, analysis of its strength and deformation characteristics, so as to take effective support form, will save a lot of resources, and take preventive measures. If the solid rock specimen is regarded as a complete rock mass, then the excavated rock mass can be modeled with the hollow specimen. However, the current research results are few [1-5]. The marble with relatively homogeneous texture is tested in the paper, so as to study the strength, deformation and failure characteristics of the hollow specimens by combining the theory of elastic mechanics with the thick walled cylinder theory and the theory of rock mechanical strength, which provides basic data for rock mass excavation engineering.

\section{Theoretical analysis}

\section{Uniaxial compression}

Without considering the effect of interfacial friction under uniaxial compression, uniaxial stress of rock specimen bearing uniform, specimens of each point in the uniform stress, so have the same axial deformation, until it reaches the ultimate bearing material, the whole specimen hollow synchronization failure occurs mainly as brittle materials, integral tension shear failure. Ideally, the existence of circular channels does not affect the uniaxial compressive strength of specimens.

\section{Pseudo-triaxial compression}

The natural rock mass is located at a certain depth of the ground, and it is in the three direction stress state due to the combined action of the gravity stress and the tectonic stress. Therefore, the influence of the pore path on the rock mechanical properties in the presence of confining pressure should be considered. Without considering the effect of end friction, the intact rock specimen is compressed under confining pressure, and the stress at each point is uniform, and shear failure follows the mohr-coulomb criterion. But the excavation of the hollow changes the stress distribution of the 
rock specimen under the three axial compression condition, under the linear elastic state, the internal stress of specimen can be based on the plane problem of thick wall cylinder was analyzed [6]. The external pressure is $\mathrm{p}$, the internal pore pressure distribution is 0 . The radial stress and the tangential stress on the cross section of the specimen are expressed as eq.1.

$$
\sigma_{r}=\frac{R^{2} P}{R^{2}-r_{0}^{2}}\left(1-\frac{r_{0}^{2}}{r^{2}}\right)=\frac{P}{1-\eta^{2}}\left(1-\frac{r_{0}^{2}}{r^{2}}\right) \quad \sigma_{\theta}=\frac{R^{2} P}{R^{2}-r_{0}^{2}}\left(1+\frac{r_{0}^{2}}{r^{2}}\right)=\frac{P}{1-\eta^{2}}\left(1+\frac{r_{0}^{2}}{r^{2}}\right)
$$

$\mathrm{R}$ : specimen radius. $\mathrm{r}_{0}$ : pore radius. $\eta=\mathrm{r}_{0} / \mathrm{R}$ : radius ratio.

$$
\text { If } \mathrm{r}=\mathrm{r}_{0}: \quad \sigma_{r}=0, \quad \sigma_{\theta}=\frac{2 P}{1-\eta^{2}} ; \quad \text { If } \mathrm{r}=\mathrm{R}: \quad \sigma_{r}=P, \quad \sigma_{\theta}=\frac{1+\eta^{2}}{1-\eta^{2}} P
$$

The strength of rock specimens meets the coulomb strength criterion, and the intermediate principal stress $\sigma_{2}$ has no effect on the strength.

\section{Test summary}

The marble used in this test is from henan province of china. Its texture is uniform and without visible defects. The grain size of marble is $2 \mathrm{~mm} \sim 3 \mathrm{~mm}$, and the crystallization degree is relatively high and relatively fresh. Specimens machined of $100 \mathrm{~mm}$ in length $50 \mathrm{~mm}$ in outer diameter. The diameter of inner hole of hollow specimen is $10 \mathrm{~mm}$.

(1) Measure the height, diameter and ultrasonic longitudinal wave velocity of the specimens.

(2) Use servo-controlled rock testing machine carry out uniaxial compression and pseudo-triaxial compression tests on solid and hollow cylinder specimens without inner pressure.

\section{Test results and analysis}

\section{Uniaxial compression test}

Uniaxial compression test results of solid and hollow specimens are shown in table 1. Complete stress-strain curves of solid and hollow specimens are shown in fig 1 (Z: medium crystal solid specimen; ZK: medium crystal hollow specimen ).

Table 1 Uniaxial compression test results of solid and hollow specimens

\begin{tabular}{ccccccccc}
\hline \multirow{2}{*}{ Specimen number } & $D$ & $d$ & $L$ & $V_{\mathrm{P}}$ & $E_{\mathrm{av}}$ & $E_{50}$ & $\sigma_{\mathrm{s}}$ & $\varepsilon_{0}$ \\
& $/ \mathrm{mm}$ & $/ \mathrm{mm}$ & $/ \mathrm{mm}$ & $/(\mathrm{m} / \mathrm{s})$ & $/ \mathrm{GPa}$ & $/ \mathrm{GPa}$ & $/ \mathrm{MPa}$ & $/ \mathrm{mm}$ \\
\hline Z-1 & 48.7 & 0 & 98.8 & 4820 & 48.7 & 65.8 & 63.2 & 0.15 \\
Z-2 & 48.7 & 0 & 99.7 & 4911 & 47.3 & 56.6 & 62.7 & 0.16 \\
ZK-1 & 48.7 & 10 & 100.3 & 4286 & 45.9 & 48.8 & 62.6 & 0.18 \\
ZK-2 & 48.7 & 10 & 100.2 & 4514 & 46.1 & 52.1 & 63.3 & 0.18 \\
\hline
\end{tabular}

$D$-outer diameter; $d$-hollow diameter; $L$-length; $V_{\mathrm{P}}$-velocity of longitudinal wave;

$E_{\mathrm{av}}-$ average modulus; $E_{50}$-deformation modulus; $\sigma_{\mathrm{s}}$-strength; $\varepsilon_{0}$-peak deformation. 


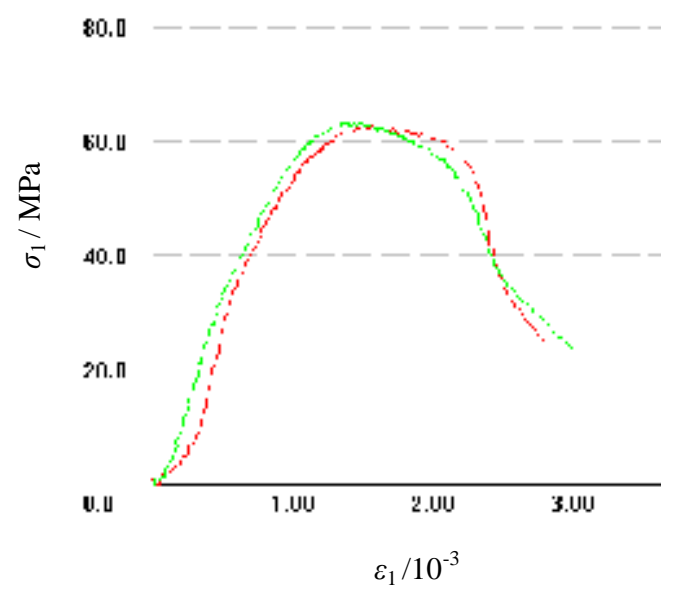

A: Solid specimens

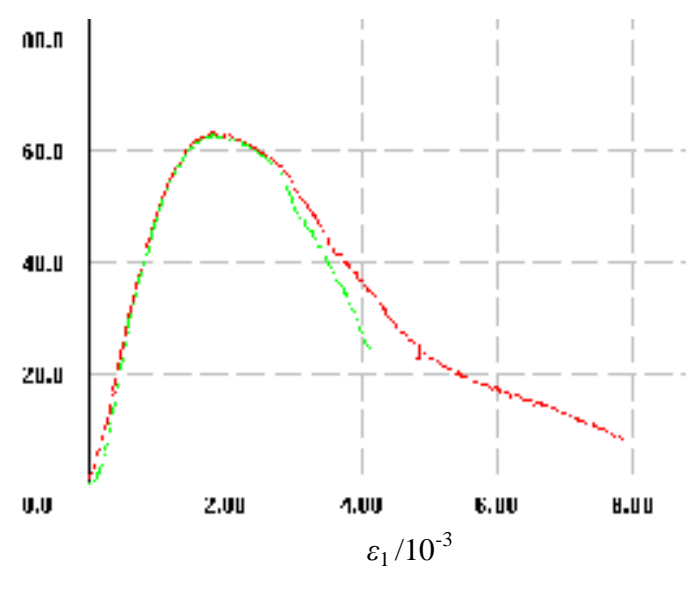

B: Hollow specimens

Fig.1 Complete stress-strain curves of solid and hollow specimens under uniaxial compression

Analysis of test results revealed:

(1) Hollow marble specimens ultrasonic velocity is $4400 \mathrm{~m} / \mathrm{s}$, compared to the solid specimen decreased by $9.6 \%$. The possible cause is that the drilling process creates new fractures within the rock. Because of the existence of the center hole in the specimen cross section, the ultrasonic velocity of the specimen will be in error. The uniformity and thickness of the applied mixture during testing of the ultrasonic velocity also affect the test results, so the ultrasonic velocity data only qualitatively explains the uniformity of the specimen material.

(2) The young's modulus is an important parameter to reflect the rock properties. Medium crystal hollow marble specimens with average elastic modulus relative to the solid specimen decreased $4.2 \%$, which is not significantly decreased. The existence of the hollow does not affect the stress distribution of the points in the specimen theoretically and does not change the young's modulus of the rock, the decrease of young's modulus of the rock may be the result of the interaction between the dispersion of experimental results and the fracture produced by specimen processing, because the longitudinal wave velocity of the solid specimen is slightly higher than that of the hollow specimen, there are more internal cracks in the hollow specimen.

(3) The average uniaxial compression strength of medium crystal solid marble specimens is 63.0MPa, that of hollow marble specimens is $63.0 \mathrm{MPa}$. In view of the material heterogeneity and the discrepancy of the test results, it can be concluded that the existence of the pores has no significant effect on the strength of uniaxial compression.

(4) Analysis of stress and strain curve shows that the solid specimen and the hollow specimen has a similar deformation law. The existence of the hollow has little influence on the uniaxial compressive strength and deformation of the rock, and the internal stress of the specimens tend to be uniformly distributed, resulting in a single section shear slip failure, which is no significant difference from that of solid specimens. Failure patterns of hollow specimens under uniaxial compression are shown in fig 2 . The whole specimen is subjected to uniform uniaxial stress, and the inner wall of the hollow is not damaged first when the material bearing limit is reached. Therefore, the existence of the hollow does not change the damage form of the rock specimen.

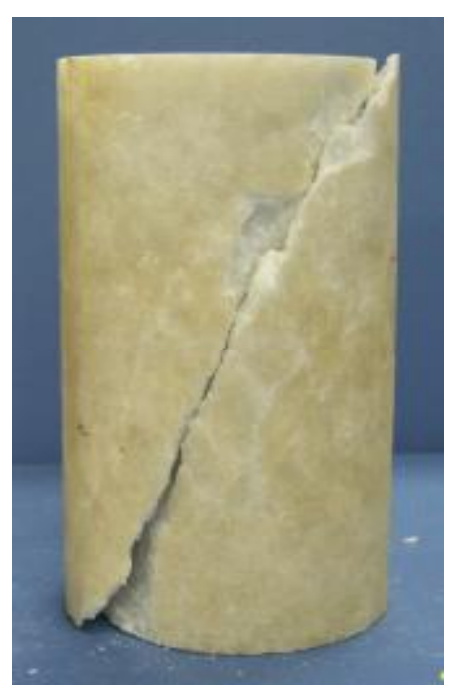

Fig.2 Failure patterns of hollow specimens 


\section{Pseudo-triaxial compression test}

Pseudo-triaxial compression tests results are shown in table 2, Stress-strain curves of solid and hollow specimens are shown in fig 2.

Table 2 Test results of pseudo-triaxial compression

\begin{tabular}{cccccccccc}
\hline \multirow{2}{*}{ Specimen number } & $D$ & $d$ & $L$ & $V_{\mathrm{P}}$ & $\sigma_{3}$ & $E_{\mathrm{av}}$ & $E_{50}$ & $\sigma_{\mathrm{s}}$ & $\varepsilon_{0}$ \\
& $/ \mathrm{mm}$ & $/ \mathrm{mm}$ & $/ \mathrm{mm}$ & $/(\mathrm{m} / \mathrm{s})$ & $/ \mathrm{MPa}$ & $/ \mathrm{GPa}$ & $/ \mathrm{GPa}$ & $/ \mathrm{MPa}$ & $/ \mathrm{mm}$ \\
\hline Z-3 & 48.8 & 0 & 100.0 & 4545 & 5 & 45.9 & 31.5 & 81.2 & 0.36 \\
Z-4 & 48.7 & 0 & 100.5 & 4389 & 10 & 52.8 & 43.3 & 97.8 & 0.65 \\
Z-5 & 48.7 & 0 & 100.3 & 4478 & 20 & 48.9 & 44.1 & 131.6 & 1.02 \\
Z-9 & 48.7 & 0 & 99.9 & 4541 & 30 & 49.5 & 36.7 & 162.7 & 1.53 \\
Z-6 & 48.7 & 0 & 98.1 & 4671 & 40 & 53.2 & 51.0 & 193.0 & 2.30 \\
ZK10-3 & 48.7 & 10 & 99.1 & 4484 & 5 & 45.1 & 43.2 & 82.1 & 0.29 \\
ZK10-6 & 48.7 & 10 & 100.4 & 4502 & 10 & 52.3 & 52.4 & 99.9 & 0.45 \\
ZK10-5 & 48.7 & 10 & 100.3 & 4438 & 20 & 50.3 & 53.0 & 132.1 & 0.85 \\
ZK10-7 & 48.7 & 10 & 100.6 & 4245 & 30 & 50.6 & 54.9 & 161.3 & 0.96 \\
ZK10-4 & 48.7 & 10 & 100.7 & 4267 & 40 & 52.9 & 50.1 & 187.3 & 1.18 \\
\hline
\end{tabular}

Analysis of test results revealed:

(1) The effect of confining pressure on young's modulus of different grain marble is different. With the increase of confining pressure, it is helpful to the closure of the rock specimen, and the amount of slip caused by the internal crack is controlled with the increase of the confining pressure, and the elastic modulus is improved. The increase of confining pressure has no significant effect on the young's modulus of the medium crystal marble, which may be due to the low internal strength and relatively high strength.

(2) Due to the existence of the hollow to increase the specimen a free surface, in the presence of confining pressure, the specimen internal stress is no longer evenly distributed, the surface of the tunnel is subject only to axial stress and tangential stress, but the total axial deformation does not change. Therefore, the material near the tunnel near the confining pressure will first destroy and the hollow collapse, the remaining part continues to carry, under the same load the specimen's bearing area is reduced, so its carrying capacity will be reduced. By observing the hollow specimens after the test, the greater the confining pressure, the more serious the collapse of the channel, the more the loss of the bond force. Analysis of the compression curve shows that after the bearing peak, although the hollow specimen compared with the solid specimen was dropped, but the intensity difference is not particularly large, because the failure of hollow specimen begin from the hollow to gradually destroy, but the hollow will not be unlimited expansion, other parts of the specimen shear slip destruction occurred under the action of the cohesive force and friction.

(3) Under the same confining pressure and loading conditions, the peak deformation of the hollow specimen is obviously lower than that of the solid specimen, especially under the high confining pressure, when the confining pressure is $40 \mathrm{MPa}$, the peak deformation of the medium crystal marble is reduced by $49 \%$. Fig 3 shows that the existence of the hollow has little significantly affect to the 
deformation of the rock in the pre-peak stage, no matter how the confining pressure changes, that's because the specimen did not produce a large plastic deformation at the beginning of the loading, and the hollow did not rupture. With the increase of axial stress, plastic deformation occurs in rock specimens, the inner wall of the pore aperture near the material yield first, gradually expand the other part to load, the effective bearing area but continued to decline, which leads to the gradual failure of the whole yielding under the condition of small strain in the axial direction, which may be the main reason for the peak deformation of the hollow specimen lower than that of the solid specimen. The existence of the hollow accelerates the weakening of the specimen, and the greater the confining pressure, the greater the influence of the hollow on the deformation.
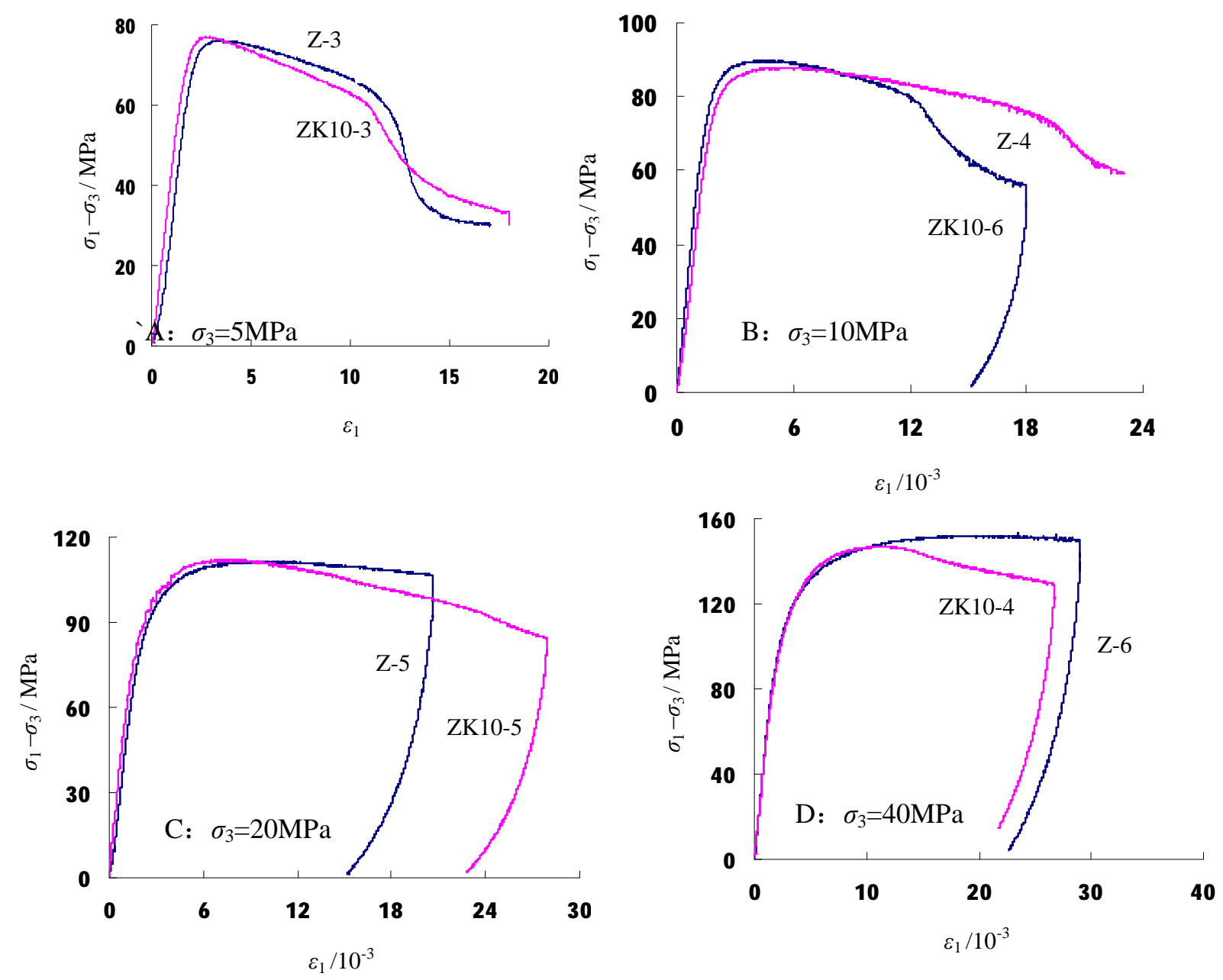

Fig.3 Stress-strain curves of solid and hollow specimens under seudo-triaxial compression

(4) Under the combined action of axial compression and confining pressure, the whole specimen is subjected to an overall shear sliding failure. While, a free surface is added to the hollow specimen inside the hollow so that the forces at each point of the specimen are different, the closer to the hollow wall, the smaller the radial stress, and the inner wall of the hollow is only subjected to axial and tangential stresses. During the test, the confining pressure is first loaded, then the axial pressure is gradually loaded, and the same axial deformation occurs at each point of the specimen, the material near the inner wall of the hollow first yields, the pore failure, and the pore size gradually increases, the other parts of the specimen continue to bear under the action of cohesive force and frictional force, and undergo shear slip failure until the whole specimen is yielding and has a stepped failure surface. With the increase of confining pressure, the ladder is more and more obvious, and the specimen has obvious hole collapse phenomenon. 


\section{Conclusion}

(1) The existence of the hollow does not affect the strength, deformation and failure characteristics of the rock under uniaxial compression.

(2) Under pseudo-triaxial compression, the strength of the hollow specimen increases linearly with the confining pressure. The strength of hollow specimens are not very different from that of solid specimens under low confining pressure, even slightly larger than that of intact specimens. When the confining pressure is $40 \mathrm{MPa}$, the strength of the hollow specimen is obviously lower than that of the intact specimen, and the young's modulus is less sensitive to the pore and confining pressure.

(3) The peak deformation of the hollow specimen is obviously lower than that of the solid specimen under pseudo-triaxial compression. The existence of the hollow accelerates the weakening of the specimen, and the greater the confining pressure, the greater the influence of the hollow on the deformation. Under the combined action of axial compression and confining pressure, the whole specimen is subjected to an overall shear sliding failure. While, a free surface is added to the hollow specimen inside the hollow so that the forces at each point of the specimen are different, the closer to the hollow wall, the smaller the radial stress, and the inner wall of the hollow is only subjected to axial and tangential stresses. The material near the inner wall of the hollow first yields, the pore failure, and the pore size gradually increases, the other parts of the specimen continue to bear under the action of cohesive force and frictional force, and undergo shear slip failure until the whole specimen is yielding and has a stepped failure surface. With the increase of confining pressure, the ladder is more and more obvious, and the specimen has obvious hole collapse phenomenon.

\section{Acknowledgements}

This work was financially supported by the educational commission of hubei province of china

(B2017257), youth research project of hubei polytechnic university of china (08yjz15Q). Thanks to the experimental support provided by henan polytechnic university and the guidance of professor you mingqing and professor su chengdong.

\section{References}

[1] Talesnick M L, etc. Completing the hollow cylinder methodology for testing of transversely isotropic rocks: torsion testing. International Journal of Rock Mechanics and Mining Sciences, 1999, 36(5): p.627

[2] You Mingqing, etc. Experimental study on strength and deformation properties of hollow cylindrical specimens of marbles. Chinese Journal of Rock Mechanics and Engineering, 2007, 26(12): p.2420

[3] LABIOUSE V, etc. Laboratory and in situ simulation tests of the excavation damaged zone around galleries in Opalinus clay. Rock Mechanics and Rock Engineering, 2014， 47(1): p.57

[4] YOU Mingqing, etc. Study of strength and failure of hollow cylinders and rings of sandstone under compression-tension stresses. Chinese Journal of Rock Mechanics and Engineering, 2010, 29(6): p.1096

[5] SU Chengdong, etc.. Experimental study on deformation and strength properties of hollow cylindrical specimens of limestone under compression. Chinese Journal of Rock Mechanics and Engineering, 2016, 35(Supp.1): p.2739

[6] XU Zhilun. Elasticity meehanics(Vol. 1). Higher Educafion Press, 1979 